\title{
Die unheilbaren Harnblasenfisteln am Bauch der Greise.
}

\section{Ein Beitrag zur Werthschätzung der Castration.}

(Vortrag mit Demonstration gehalten am 14. December 1896 in der freien Vereinigung der Chirurgen Berlins.)

Von

Prof. Dr. Edm. Rose.

Meine Herren! Jedem beschäftigten Praktiker sind wohl solche Fälle bekannt, dass Harnblasenfisteln am Bauch alter Männer allen Heilversuchen widerstanden haben, obgleich sie eigentlich gerade unter allen Harnfisteln am leichtesten heilen sollten, weil sie doch offen zu Tage liegen. Wie oft sieht man solche Fälle!

Lange Jahrzehnte hat man sich bemüht, ehe man seit Dieffenbachs ersten Erfolgen 1) 1836 die Unheilbarkeit der Blasenscheidenfistel zu uberwinden lernte. Wie vicle Schwierigkeiten haben sich danach noch bei den seltenen Fällen von Harnröhrenblasenscheidenfisteln geboten, wenn wirklich ein Mangel des ganzen Schliessmuskels vorlag.2) Welche grossen Hindernisse bieten auch heute noch itrer tiefen Lage wegen die Blasenmutterfisteln dar! Niemand war das auffallend, der es kennt, wie eng bei solehen Fällen oft das kleine Becken ist, wie diese Blasenfisteln oft unseren Instrumenten kaum zugänglich sind. Ist es da nun nicht uberraschend, dass gerade Blasenfisteln am Bauch, die ganz offen vor uns liegen und unserer Technik deshalb gar keine Schwierigkeit bieten sollten, manchmal allen bekannten Operationsmethoden den hartnäckigsten Widerstand leisten? Noch dazu sind das dieselben Fisteln, die man in anderen

1) Vgl. Edmund Rose: „Die Operation der Blasenscheidenfistel“, S. 81 in den älteren Charité-Annalen Bd. XL. Berlin 1863.

2) Vgl. Edm. Rose : „Ueber den plastischen Ersatz der weiblichen Harnröhre“. Vortrag auf der Versammlung der Naturforscher und Aerzte in München den 19. Sept. 1877. Deutsche Zeitschrift für Chirurgie. Bd. IX, S. 122 und Fdm. Ros e: Therapeutische Monatshefte. 1887. Nov. Ueber die Anlage einer Harnröhreim Mastdarm und die Schwierigkeiten dieser Operation. 
Fällen fast ganz von selbst und anscheinend ohne unser Zuthun schnell verheilen sieht.

Vom Lande kommt ein Herr, dem wegen Harnverhaltung bei impermeablen Stricturen der Blasenstich gemacht war. Kunstgerecht wird ihm der äussere Harnröhrenschnitt gemacht, die Cantule am Bauch entfernt, und ohne dass man sich um seine Blasenfistel recht gektimmert hat, ist sie schon fest und dauerhaft geschlossen, ehe noch die Urethrotomiewunde ganz vernarbt.

Ein grosser Junge wird in das Krankenhaus gebracht mit einer Fleurant'schen Cantlle in der Blase, dessen Damm infolge eines Sturzes nichts wie eine dicke Narbenmasse darbot. Da gleich hinter dem Scrotum jede Spur einer Harnröhre aufhörte, machte ich mit Benutzung der Fistel eine Cystostomie, führte von der Blase aus einen silbernen Katbeter in den hinteren Rest der Harnröhre und verband diesen dann einfach aussen durch den Schnitt mit den Resten der Harnröhre am Scrotaltheil des Penis. Während sich dann in gewöhnlicher Weise um den Katheter eine neue Harnröhre bildete, heilte die Harnfistel am Bauch von selbst.

Sehen wir uns bei diesem Gegensatz nach den näheren Umständen um, so verräth sich zunächst fur diese Verschiedenheit im Verlauf als Ursache das Greisenalter. Die Leute, welche wir mit unheilbaren Bauchfisteln herumgehen sehen, sind entweder ältere Steinkranke, denen der hohe Steinsehnitt gemacht ist; noch nach Jahr und Tag ist manchmal die Wunde nicht geheilt. Oder es sind Prostatakranke, denen man die Paracentesis vesicae wegen Harnverhaltung gemacht bat. Die F leurant'sche Canüle sind sie los, aber nicht ihre lästige Harnfistel am Bauch, die das Leiden des "Prostatismus" noch verschlimmert.

Dass bei Greisen Wunden durch Eiterungen manchmal sehr langsam, meist langsamer heilen, als bei jungen Leuten, ist ja bekannt; allein schliesslich bringt man ja aueh bei ihnen die grössten Wunden zum Verschluss. Das kann also nicht der Grund sein! Der wahre Grund ist die Harnstanung, wie sie die Prostataschwellung der Greise mit sich führt.

Damit ist die Heilung dieser unheilbaren Fisteln gegeben.

Einen charakteristischen Fall der Art wollte ich ihnen bier vorstellen.

Der 64 Jahre alte pockennarbige Arbeiter Wilhelm H., geboren in Senzig, wurde aus Königswusterhausen wegen Urinbeschwerden nach Bethanien geschickt, in dem schon seine Frau in Heilung an einem umfangreichen Brust- und Achseldrüsenkrebs lag. 
So kräftig und gesund sie sonst aussah, hatte sie doch in ihrem langen Ehestande keine Kinder bekommen. Seit mehreren Jahren litt der Mann an wachsenden Harnbeschwerden, die sich zeitweise bis zur Verbaltung steigerten. Anfangs wollte er erst Nichts von seinen Trippern wissen; unter uns bat er mir später zugegeben, vor langer Zeit in seiner Jugend daran gelitten zu baben. Am Damm war er mit Narben bedeckt, die von Geschwtiren herribrten, welche er nicht weiter beachtet hatte. Er wurde am 4. Januar 1895 (unter Journalnummer 141) in die chirurgische Abtheilung aufgenommen.

Nach den nöthigen Vorbereitungen am 9. Januar narkotisirt, ergab sich bei der Untersuchung, dass die Harnröhre so mit Stricturen besetzt, dass nur die feinste geknöpfte Roser'sche Sonde sich durch den Penis durchfthren liess; die Untersuchung vom Mastdarm ergab ausserdem eine harte derbe Vergrösserung der Prostata. Vorläufig stand ich von Weiterem ab, allein trotz Bettruhe, Bädern und dergleichen erfolgte schon am Abend ein Schüttelfrost, der sich am 11. Januar Abends mit vollständiger Harnverhaltung wiederholte. Mein ältester Assistent versuchte in der Chloroformnarkose den Katheterismus, allein die Harnröhre war impermeabel geworden. Unter erschwerten Umständen (durch zufällige Concurrenz von Urlaub und Krankheit - war den Abend ein Mangel von Wärtern und Assistenten) habe ich ihm dann in der Nacht durch die Narbenmasse den äusseren Harnröhrenschnitt gemacht. Allein auch von der Wunde aus liess sich der Katheter nicht gleich in die Blase einftihren. Unter diesen Umständen machte ich kurzen Process, fugte die Cystostomie hinzu, überzeugte mich beim Eingehen mit dem Finger in die Blase, dass der mittlere Lappen der allseitig geschwollenen Prostata haselnussgross in die Harnblase hineinreichte, und fügte dann leicht mittelst retrogradem Katheterismus einen Dauerkatheter durch die Dammwunde hinaus.

Am 19. Januar wurde dann in der Narkose ein Dauerkatheter von der Eichel aus in die Blase gefubrt.

Am 15. März war die Uretbrotomiewunde geheilt, aber nicht die Cystostomia suprapubica, trotzdem der Dauerkatheter, soweit der Blasenkatarrh es zuliess, ständig für freien Abfluss sorgte und immer wieder erneuert worden war. Allmählich verwandelte sich der hohe Blasenschnitt in eine Fistel, ein Zustand, der ja jetzt von vielen Operateuren bei der Prostatahypertrophie als palliative Heilung gewinscht und verberrlicht wird, mir aber doch stets sehr beklagenswerth erschienen ist.

H. war derselben Ansicht uiber diese Infirmität! 
Am 24. April wurde nach anderweitigen Versuchen deshalb die Fistel mit dem Gltheisen in der Narkose zerstört und immer wieder mit Dauerkatheter, Wasserleitung und Blasenausspullungen aller Art nachgegeholfen. Allein Alles vergeblich!

Am 2. Mai gab es in der Urethrotomienarbe einen kleinen Abscess, der am 20. Mai wieder geheilt war.

Einmal habe ich auch die Hantdecken von der Fistel weit abgelöst und die Wunde mit Nähten geschlossen. Wieder ohne bleibenden Erfolg!

Als ich einmal am 6. Juni einen Nélaton als Dauerkatheter eingelegt, wurde er sogar in der Nacht durch die Bauchwunde herausgepresst. Kurz, nichts wollte helfen!

Da haben wir uns denn Beide zu seiner Castration (Orchidectomia duplex) entschlossen, die am 18. Juni in der Narkose mit gleichzeitiger Cauterisation der Fistel vorgenommen wurde.

Am 10. Juli waren alle Wunden sicher geheilt.

Am 15. Juli ist er zuerst aufgestanden und ging am 30. Juli gesund nach Hause.

Wie jeder Urethrotomirte sollte er sich dann zur Controle seiner Harnröhrenlichtung zeitweilig wieder vorstellen. Bei dieser Gelegenheit baben wir jedesmal nach Einführen eines dicken silbernen Katheters vom sorgfältig gereinigten Mastdarm aus das Verhalten der Prostata verfolgt. Nach einigen Monaten hatte der Finger beim Tasten nach dem Verlauf des Katheters das Gefubl, als ob er über ein halbvolles Wasserkissen streiche; deutlich konnte man aber noch die Contouren der Prostata in ibrem alten Umfang bemerken. Heute Morgen dagegen (14. Dec.) lag der Katheter in ganzer Länge wie nackt da, nur von der Mastdarmwand bedeckt. Nicht eine Spur von Prostata liess sich mehr entdecken! Der Kranke hat sich von seinem Leiden ganz erholt, ist stark geworden und hat seinen Bart behalten, soviel er davon hatte. Auf meinen Wunsch hat er dann tüchtig Wasser getrunken. Als er es gar nicht mehr halten konnte, entleerte er weit über ein Liter in vollem Strahl. Der Harn ist eiweissfrei und schon lange klar. So ist er also frei von allen Beschwerden.

Wir haben hier also durch Atrophie einen künstlichen Defect der Prostata vor uns, der an Gründlichkeit wetteifern kann mit dem congenitalen und dem senilen Defect, wie sie zuweilen vorkommen, wie ich sie frlber mehrmals in meiner Klinik in Zürich meinen $\mathrm{Zu}$ hörern habe demonstriren können. Während dieser allmählichen Atrophie der Prostata hat sich die anscheinend unheilbare Blasenfistel obne Weiteres geschlossen.

Dieser Fall ist ausserordentlich lehrreich, weil ich die Prostata 
vor der Castration sowohl vom Rectum, wie von der Blase aus mit dem Finger controlirt habe und nach derselben ihren allmählichen Schwund verfolgen konnte. Dabei möchte ich nochmals darauf aufmerksam machen, dass die Schwellung und Prominenz ihres mittleren Lappens vor der Operation constatirt worden ist, und dass es sich um eine harte Prostatahypertrophie in unserem Fall gehandelt hat.

Die Function der Harnorgane ist jetzt so gut, dass sie nicht besser denkbar ist.

Und nicht blos die durch die Prostatahypertrophie mitbedingten jahrelangen Harnbeschwerden sind vollständig gehoben, sondern auch die Prostata ist vollständig verschwunden, und mit diesem Hinderniss der Excretion ist schnell die früher unheilbare Blasenbauchfistel glatt geheilt.

So hat uns hier die Castration einen wahrhaft uberraschenden Erfolg ergeben, der für diese Errungenschaft unseres Jahrhunderts ganz begeistern muss.

Wenn man Epididymiscysten und Spermatocelen alter Leute operirt, so habe ich mich uberzeugt, dass selbst in sehr hohem Greisenalter die Samenfäden wohl erhalten sein können und sich im frischen Präparat manchmal ganz ausserordentlich kräftig bewegen. ${ }^{1 j}$ Dass die Grösse und Function der Prostata von der Function der Hoden abbängt, ist längst offenbar, und scheint mir das beiläufig auch für andere Krankheiten nicht ohne Bedeutung.

Als Contraindication der Castration bei Hodentuberculose hat man den Blasenkatarrh und die Prostataanschwellung aufgestellt. Man ist der Meinung, dass sie stets ein Beweis für das Fortschreiten der Tuberculose durch die Samenwege auf die Harnwege sind, und damit die Entfernung der Hoden nutzlos, wenn nicht schädlich sei. Das ist eine grosse Thorheit! Dadurch allein sollte man sich nicht unter allen Verbältnissen von einer manchmal so ausserordentlich dankbaren Operation abhalten lassen, wie die Castration bei echter Hodentuberculose sein kann.

Schon vor Jahrzehnten habe ich einen bescheidenen Commis aus Einsiedeln einen Hoden wegen käsiger Tuberculose der Epididy-

1) Ein Beispiel bot der 70 Jahre alte Particulier Ernst Z., der am 16. Januar 1896 in der 2. Klasse meiner Station in Bethanien mit Spermatocele rechts eintrat. Am 17. machte ich ihm, weil er einen Schlaganfall überstanden, anstatt der Narkose nach der Abzapfung erst eine Cocaininjection in die Cyste und nach ihrer Entleerung die übliche Jodinjection ganz schmerzlos. Er hefand sich danach so wohl, dass er sich am 19. Januar nicht mehr im Hause halten liess, und ist geheilt. Entleert wurde ein halber Liter Flüssigkeit, in der sich ausserordentlich zahlreiche, wie tobsüchtig sich bewegende Samenfäden trotz der 70 Jahre fanden.

Deutsche Zeitschrift f. Chirurgie. XLVI. Bd. 
mis und disseminirter Miliartuberculose des Hodens selbst entfernt. (Die Diagnose lag auf der Hand und ist auch im Curs der pathologischen Anatomie am Präparat anerkannt worden.) Nach langen Jahren war ich sehr erstaunt, als mich derselbe auf der Durchreise, jetzt ein behäbiger Herr in prachtrollem Pelz, mit einer netten jungen Frau wegen einer Kleinigkeit an seinen Geschlechtstheilen consultirte. Beiläufig batten sie Kinder beiderlei Geschlechtes, und die ängstlich besorgte junge Frau demonstrirte mir mit, was ihr am Scrotum nicht ganz geheuer schien.

So babe ich denn nach dieser Erfahrung im Anfang der 80iger Jahre ') einen Offizier, der schon seit Jahren mit einer schönen jungen Frau kinderlos lebte, ruhig operirt, und das, trotzdem er sehr an Blasenkatarrh litt. Der Befund war derselbe: käsige fistulöse Tuberculose des Nebenhodens und miliare im Hoden. Die Heilung hat sich etwas (1 Monat ungefähr) verzögert, allein der hartnäckige Blasenkatarrh verschwand damit. Auch hier ist dann später zur grossen Freude ein Kind ersebienen, und der Herr, welcher jetzt im besten Wohlsein nach anderthalb Decennien in hober Stellung mit einem leichteren Posten versorgt ist, hat mir noch jungst durch einen Collegen von seinem Wohlbefinden Mittheilung machen lassen.

Ich fubre das gern auch deshalb an, weil noch jüngst kurz vor seinem Tode Lewin behauptet hat, die Hodentuberculose sei uberhaupt kein Object für die Chirurgie!

So habe ich mich auch noch jungst im Spital überzeugen können, dass, wenn sich bei einseitiger Orchidectomie die Heilung infolge älterer organischer Fehler verzögert schon im Krankenbaus eine Abnahme der betreffenden Prostatahälfte in zweifelloser Weise stattfand.

Der 64 Jahr alte Arbeiter Karl St. aus Rixdorf wurde am 11. Norember 1895 auf die chirurgische Station in Bethanien verlegt von der inneren, auf der er wegen eines Herzfehlers sich befunden hatte. Wegen Tuberculosis testis sinistri wurde am 13. November (mit Chloroform 10,0) die Orchidectomie gemacht, welche $z$ war glatt und ohne weitere Zufälle, aber unter diesen Verhältnissen ausserordentlich langsam heilte. Bei einer Untersuchung in der Narkose am 19. Februar 1896 war die entsprechende Seite der Vorsteherdrüse besonders stark verkleinert, gegenüber der anderen Seite und dem fruheren Befunde.

Am 28. März wurde er geheilt entlassen.

Ich bedaure, dass ich auf diese Consequenz der Orchidectomie früher nie weiter geachtet habe.

So bin ich denn der Meinung, jeder Prostatiker, der dauernd aum Katheter verurtheilt ist, oder der gleichzeitig, wie das dabei so oft

1) Den 21. August 1881. 
der Fall ist, an Blasenstein leidet, jeder Prostatiker, der schon eine künstliche Bauchfistel davon getragen hat oder wegen falscher Wege oder auch aus anderen Gründen doch dort an Ort und Stelle operirt werden muss, sollte sich in derselben Sitzung gleich castriren lassen. Es ist zu bedauern, dass man dabei noch auf Widerstand stösst.

Wie oft werden noch, das sieht man bei Consultationen, selbst von guten Aerzten und alten Praktikern, wenigstens noch die tertiären und secundären Gefahren der Prostatahypertrophie - der ganze Jammer des jetzt sogenannten „Prostatismus" unterschätzt oder wohl gar übersehen! Wie oft geben Greise mit sogenanntem Residualurin an chronischer Urämie mit ihren Schleimhautkatarrhen unter der Diagnose „chronischer Magenkatarrh" oder asthmatische Beschwerden zu Grunde, Männer, deren Beschwerden sich durch eine geordnete Localtherapie oft förmlich abschneiden lassen. Das „Katheterleben" ist dann oft die vorläufige Rettung, aber doch nur eine gar zu traurige. Die meisten älteren Leute werden dadurch mindestens ganz abbängig von ihrer Umgebung und von der Zartheit ihrer Hülfsleistung. In der consultativen Privatpraxis habe ich freilich Kranke, die schon seit Tagen, weil man sie bei voller Blase nicht katheterisiren konnte, in tiefem urämischen Coma lagen, durch Einlage eines Dauerkatheters gerettet. Allein 10 Jahre später sind sie trotz der allerbesten Verhältnisse doch an Nierenschrumpfung infolge des Reizes des täglichen Katheterns zu Grunde gegangen. Die armen Kranken dagegen, die nach Bethanien kommen, sind selten so gut daran! Da kommen sie nach langen Leiden oft, um in wenigen Tagen zu sterben, meist erst mit falschen Wegen und allen möglichen Folgeerscheinungen, secundärer Blasensteinbildung, abgerissenen Kathetern, manchmal sogar mit mephitischer Gangrän am Damm! Da sollte doch bei Zeiten die Radicalkur eine viel grössere Rolle spielen!

Nur zwischen Prostatectomie und Castration bleibt da die Wabl, beides keine aussichtsreichen Operationen, wenn die Fälle in dieser Weise ungünstig sind. Ich habe die Prostatectomia completa von der Blase, wie vom Damm aus unter solchen traurigen Verhältnissen trotzdem gemacht, in 7 Fällen, die noch die günstigsten zu sein schienen. Keiner lebt davon, wenn auch der Erste, ein Steinkranker, Anfangs einen überraschenden Erfolg gab. In seinen furchtbaren Schmerzen und schweren Leiden ging er auf die Operation, wie auf eine Art Selbstmord, mit Freuden ein. In der nächsten Woche jedoch war er schon sehr entzlickt von dem Resultat und lebte ganz auf. Bei glattem Verlauf ging er in der 4. Woche ganz plötzlich an Coma zu Grunde; beide Nieren waren auf ein Minimum geschrumpft. 
Die anderen collabirten alle fruher infolge latenter Nierenleiden! Die Castration ist viel segensreicher, weil sie ein leichter Eingriff, wenn sie prima intentione heilt.

Rathsam ist es dabei, in der Narkose mit der linken Hand den Hoden von hinten gegen die vordere Mittellinie der betreffenden Hodensackbälfte anzudrängen, an eine Stelle dort, wo sich am wenigsten Gefässenden zeigen, und ihn durch einen möglichst kleinen Schnitt mehr und mehr herauszuzwängen. Danach wird eine Nadel mit doppeltem mittelstarken festen Catgutfaden durch den Samenstrang dicht tuber dem Nebenhoden durchgestossen und der Strang nach beiden Seiten abgebunden. Der Schnitt wird mit 3 Seidennähten geschlossen, von denen die mittelste oberhalb der Catgutligaturen durch den Samenstrang geht, um so jeden todten Raum auszuschliessen.

Da der Hoden dabei gesund oder nur unwesentlich krank, kann diese Operation in 5 Tagen dauerhaft heilen, fast ohne eine sichtbare Narbe zu hinterlassen. Es ist das ein grosser Vortheil bei so ungünstigen Fällen, hängen doch die Erfolge aller dieser Operationen viel mehr oder vorzliglich davon ab, wie weit die unmittelbaren Folgen in der Prostata und Blase, die secundären in den Nieren und die tertiären in den weiteren Organen ausgebildet sind. Wir können doch leider selten wissen, wie nahe bei den chirurgischen Blasenleiden das Ende bevorsteht, zumal bei Greisen! Immer wieder muss man hier, wie bei der Lithotripsie, dem Steinschnitt, der Operation der Blasenscheidenfistel daran erinnern, wie schwer, ja unmöglich meist das Bestehen secundärer Folgen in den Nieren bei diesen Leiden zu erkennen ist. Bei den ascendirenden Leiden der chirurgischen Blasenkrankheiten ist es ja Regel, dass Eiweiss fehlt oder nicht mehr vorhanden ist, als dem Eiter entspricht, Cylinder nicht $\mathrm{da}$ sind, und sogar oft der Druckschmerz bei der Palpation der Nierengegenden, wie sonst jeder Anhaltepunkt vermisst wird. In einigen von den Krankengeschichten ist der Mangel von Druckschmerz noch ausdrücklich am Tage vor der Operation constatirt worden. So wird man denn bei den so lange maltraitirten Fällen, wie sie schliesslich in die Krankenhäuser kommen, nur zu häufig nach scheinbar gelungener Operation lunerwartet vom Ende überrascht. Durch eitrige Pyelonephritis mit und ohne Dilatation von Harnleiter und Nierenbecken, anderenfalls durch Schrumpfniere hohen Grades mit ihren Folgen auf Hirn,| Herz und Lunge erklären die Obductionen dann leicht den uberraschenden Ausgang; er war uns stets ïberraschend, weil man zwar immer bei so ungünstigen Fällen 
darauf gefasst sein muss, ohne jedoch im Einzelfall bestimmen zu können, wie weit diese organischen Veränderungen bei ihrer Latenz schon vorgeschritten. So spielt denn Collaps und Erschöpfung erfahrungsgemäss eine grosse Rolle bei solchen Fällen, mag man sie operiren oder aucb nicht.

So kam noch jüngst in unsere Behandlung der 68jährige Wilhelm S. wegen Harnverhaltung bei Prostatahypertrophie (aufgenommen 1896 den 1. Januar. Journalnummer 128). Da er die Castration verweigerte, wurde er nur regelmässig und leicht katheterisirt. Er starb schon an 6 . Januar benommen entweder durch Erschöpfung oder Urämie; die Seetion wurde leider verweigert.

So kam der 74jährige Carl N. am 2. März 1896 in unsere Bebandlung (J.-Nr. 350) wegen Prostatahypertrophie und Blasenkatarrh und starb schon am 5. an chronischer Urämie infolge linksseitiger Pyelonephritis mit Dilatation des linken Ureters, ohne dass irgend eine Operation vorgenommen worden war.

So wurde der 75 Jahre alte Friedrich R. am 7 . April 1894 (unter J.-Nr. 456) wegen Prostatahypertrophie und Blasenkatarrh aufgenommen und starb schon am 12. April. Auf beiden Seiten bildeten die Nieren manusfaustgrosse Säcke infolge Dilatation des Beckens, inden das Parenchym bis auf $1 \mathrm{Cm}$. Breite geschwunden war. Im linken Nierenbecken ein grosses Blnteoagulum. Blasenwand $2 \mathrm{Cm}$. dick mit zahlreichen Divertikeln.

In demselben Jahre wurde ferner der 73 Jahre alte Ch. E. am 1. Mai (unter J.-Nr. 545) aufgenommen wegen Prostatahypertrophie mit Blasenentzlundung und falschen Wegen und starb schon am 10. Mai urämisch, ohne dass eine Section gestattet wurde.

Im Jahre 1892 wurde der 72 Jahre alte W. S. am 27. Juni (unter J.-Nr. 771) mit Blasenbeschwerde infolge Prostatahypertrophie aufgenommen und starb schon am 3. Juli. Wie sich bei der Section zeigte, war das Herz schlaff, die grossen Gefässe arteriosklerotisch; die vergrösserte Prostata im Inneren erweicht mit haselnussgrossen Abscessen bei doppelseitiger Pyonephrose. Milz matsch, Eiter in den Ureteren.

Im Jahre 1891 (J.-Nr. 1328) wurde der 67 Jahre alte Thierausstopfer Adolf L. am 5. December wegen Prostatahypertrophie mit Blasenkatarrh aufgenommen und starb schon am 12. December; die Obduction ergab Lungenemphysem mit Bronchitis, Hypertrophie des Herzens, das Herzfleisch brïchig und verfettet, Lebereirrbose, bis stecknadelkopfgrosse goldgelbe Concremente im Becken der rechten Niere, stark vergrösserte Prostata, in der stark suggillirten und an 
einer Stelle erodirten Blase mehrere bis taubeneigrosse Steine. Das Gehirn ödematös und sehr bleich.

Ich greife nur Fälle heraus aus den Jahren, die hier in Frage kommen. Leicht liesse sich sonst diese Liste noch weit ausdehnen.

So kommen also leider viele solche Todescandidaten mehr oder weniger in den letzten Zügen auf unsere Abtheilung.

Immerhin fällt bei so schwieriger Sachlage die Schwere der Operation doch ins Gewicht und wird die leichtere vorzuziehen sein. Alle meine Prostatectomien sind zwar örtlich gut und ohne Folgen für das Bauchfell, ohne Eintritt von Phlegmonen abgelaufen, allein je gründlicher sie die ganze Prostata beseitigen, desto weniger kann man einen gewissen venösen Blutverlust ganz vermeiden, desto länger wird die Heilung durch Eiterung den Kranken schwächen, während der Eingriff der Castration dagegen eben fast verschwindend sein kann.

Beide bilden ja natiirlich nur die letzte Zuflucht, wenn das $\mathrm{Ka}$ theterleben unabwendlich geworden, aber es wäre doch wünschenswerth, man intervenirte damit nicht so oft zu spät. Die Kranken sollten früher dazu kommen!

Als Belag fur das Mitgetheilte will ich in einem kurzen Auszug die Geschichte meiner Kranken skizziren.

\section{Meine Prostatectomien.}

I. Wilbelm K., Schiffer, 70 Jahrealt, aus Mittenwalde. (1892. J.-Nr. 366.) 2.-30. März 1892.

Vorausgegangen sind Masern, Scharlach, Tripper, Emphysem. Rechtes Trommelfell perforirt. Links Altersstaar.

1860 schon 1/2 Jahr lang wegen Ischurie katheterisirt. Seit $1863 \mathrm{Ab}$ gang von Blut im Urin und im Stuhl mit Stuhlzwang und Pfeifenkoth. Harn jetzt nur tropfenweise mit Blasen- und Kreuzschmerzen. Urin stinkend, blutig.

Sechs grosse Steine in der Blase und eine beträchtliche Prostatahypertrophie von $147 \mathrm{Grm}$. Gewicht machten ihm das Leben unerträglich.

Den 7. März 1892 Prostatectomia completa perinealis mit 27,5 Chloroform. Wie gewöhnlich wurde erst die Urethrotomie und von dem unteren Ende dieses Schnittes Seitenschnitte um den Schliessmuskel des Afters gemacht. Nach der möglichst stumpfen Entferuung der ganzen Prostata wird die ersten Tage ein Dauerkatheter von Metall eingelegt und die Wunde um denselben tamponirt, später offen behandelt.

Unmittelbar erfolgte kein Erbrechen, dagegen vom 9.-11. März 9 mal, $1 \mathrm{mal}$ noch am 13. März, und $4 \mathrm{mal}$ am 15. März. Wegen steter Verstopfung des Katheters wurde schon am 9 . März die Wunde offen behandelt, wobei jeder Schmerz aufhörte. Vom 16. März ab wiederholte Bäder. Der Verlauf war vollständig fieberlos, am 29. März Abends sank die Temperatur jedoch auf $35,1^{\circ}$, und der Tod erfolgte am 30 . März.

Der bei der Operation unbedentend verletzte und mit einer Naht geschlossene Mastdarm war sofort vollständig geheilt. Geringe Atheromatose 
der Aorta ascendens. Milz gross, matsch. Linke Niere unregelmässig eingesunken mit dilatirtem Becken, Ureterenschleimhaut theilweise nekrotisch. Im Nierenbecken und Ureter Steinkrtimel. - Phtisis renalis calculosa. Rechte Niere etwas vergrössert, Blase trabeculär. Bauchfell normal. Keine Nebenverletzung.

II. Friedrich K., Arbeiter, 73 Jahre alt, ans Berlin. (1892. J.-Nr. 423.) 16. März bis 1. Mai 1892.

Vorausgegangen Scharlach und Typhus.

Im November 1891 Incontinenz mit Tenesmus und schmerzhafter Retention. Prostata weich, oberer Rand nicht abreichbar. Trotz Dauerkatheter Albuminurie mit und ohne Blut.

Am 15. April beim Fortlassen absolute Retention.

Am 18. April Druckschmerz in den Nierengegenden.

Am 28. April kein Blut mehr, keine Cylinder, aber Albuminurie. Kein Fieber.

Am 30. April Exstirpatio prostatae circularis completa, trigoni et vesicularum partialis mit 12,5 Gramm Chloroform und 10 Gramm Aether.

Am 1. Mai Erbrechen, Singultus, Continenz, plötzlicher Collaps.

Milz matsch. An der Nierenoberfläche mehrere Einziehungen und Hämorrhagien. Herz sehr sehlaff. Bauchfell normal, zeine Nebenverletzungen.

IIr. Julius K., Hauseigenthumer aus Gascht in der Neumark, 72 Jahre alt. (1892. J.-Nr. 592.) 7.-17. Mai 1892.

Vor 5 Jahren wegen Brucheinklemmung operirt, bekam er Harnverhaltung. Trinker.

Kathetert sich seit 2 Jahren 2 mal täglich, zuletzt dreistindlich. Die letzten 3 Tage vergebliche Versuche mit falschen Wegen.

Urin blutig, verstopft sofort den Dauerkatheter. Obere Grenze der Prostata in der Narkose nicht abzureichen.

17. Mai Prostatectomia completa mit 16,0 Chloroform. 2 mal Erbrechen nach der Operation. Kein Fieber. Tod an Collaps.

Arteriosklerose, weiches Herzfleisch mit Dilatation, Emphysem der unteren Lungenlappen, Nierenschrumpfung. Fettleber. Taubeneigrosser Stein in der harten strahligen Gallenblase. 2 Polypen im Magen, der hämorrhagisch entzündet ist. Schädeldach und Pia verdickt. Bauchfell normal. Keine Nebenverletzungen.

IV. August K., Maureraus Berlin, $69 \mathrm{Jahre}$ alt. (1893. J.-Nr. 409.) 17.—29. März 1893.

Vorausgegangen sind Typhus, Lungenentzindung, Emphysem mit reichlichem Katarrh und Cyanose.

Blasenbeschwerden seit 2 Jahren, besonders Tenesmus. Seit 3 Tagen Retention, weshalb er draussen 1 mal katheterisirt und beim späteren Scheitern gestern und heute mit Blasenpunction behandelt ist. Hier ging der Katheterismus leicht, allein der Urin war tribb, eitrig mit Nierenbeckenepithelien.

Am 25. März 1893 Prostatectomia completa perinealis mit 12,0 Chloroform und 25,0 Aether. 
Kein Erbrechen, und nur am 22. März und vom 25. März an Abends Fieber bis $38,9^{\circ}$. Puls nie tiber 120 . R. nie tiber 24 .

27. März offene Wundbehandlung.

Tod an Lungenoedem.

Eitrige Bronchitis mit Bronchectasie. In den Lungenspitzen ausgeheilte Tuberculose. Cor bovinum; Klappen normal. Eitrige Pyelitis mit Dilatatio ureterum. Eitrige hämorrbagische Cystitis mit polypösen Wucherungen. Bauchfell normal. Keine Nebenverletzungen.

V. J.v. St., Oberstlieutenant aus Berlin, $62 \mathrm{Jabrealt.}$ (1893. J.-Nr. 1042.) 2.-11. September 1893.

Voransgegangen sind Tripper in der Jugend, Urinbeschwerden seit 20 Jahren, „Katheterleben" seit 5 Jahren. Eine Punctio suprapubica vor 5 Jahren ist seit Mai als Fistel wieder aufgebrochen, ebenso wieder jetzt am 2. August. Seit Mai Abscesse am Damm neben einer Urethrotomienarbe.

Viel Eiter im Urin, der keine Cylinder und wenig Blut enthălt. Kein Druckschmerz in der Nierengegend.

4. September. Zunächst wurde wegen Unterminirung die Blasenfistel am Bauch dilatirt.

Am 11. September Prostatectomia completa mit 12,5 Chloroform. Die Prostata enthält einen grossen Abscess. Die Umfänge des linken Lappens betrugen $11,0,12,5$ und $14,5 \mathrm{Cm}$. und des rechten $9,0,9,5$ und 9,0 .

Der Tod erfolgte bei dem anscheinend sehr kräftigen Mann 5 Stunden nach der Operation im Collaps.

Die Ureteren waren kleinfingerdick und voll Eiter, ebenso die dilatirten Nierenbecken. Cirrhose beider Nieren mit vielen bis haselnuss. grossen Abscessen. (Rechts $9 \times 4,5 \times 2,5 \mathrm{Cm}$., links $8,5 \times 4 \times 2,5 \mathrm{Cm}$.) Fettkapsel verdickt. Blase hypertrophisch, Bauchfell normal, keine Nebenverletzungen.

VI. J. W., Schneidermeister aus Oderberg, $68 \mathrm{Jahre}$ alt. (1894. J.-Nr. 291.) 17. Januar bis 22. Februar 1894.

Links Leistenbruch vor 8 Jahren wegen Einklemmung operirt. Vor 3 Jahren, nachdem mehrere Jahre blutiger Urin bestand und Schuttelfröste vorangegangen, hoher Steinschnitt. Rechts Leistenbruch durch den schweren Stuhlgang seit mehreren Monaten.

Der nach der Steinoperation zurlickgebliebene Blasenkatarrh hat jetzt 2u heftigen Schmerzen und Tenesmus geführt.

Kein Druckschmerz in der Nierengegend.

21. Februar. Prostatectomia completa mit 12,5 Chloroform und 32,5 Aether. Beide Lappen sind je uber wallnussgross (56 Gramm). Entfernt werden ausserdem ein pflaumengrosser pyramidaler Stein $(50 \mathrm{Grm}$. schwer) und ein viereckiger (39 Grm. sebwer), welcher in einem Recess hinter der Steinschnittnarbe festsass; beide baben sich um Seidennäthe gebildet.

Mehrmaliges Erbrechen hei grosser Schwäche.

22. Februar Abends Collaps.

Im rechten Oberlappen mehrere verkalkte, erbsengrosse Herde, Lungen- 
oedem. Geringe Arteriosklerose der Aorta. Braune Atrophie des Herzen. Perisplenitis calculosa. Linke Niere mit hullnereigrosser Cyste, rechts in der Rinde haselnussgrosser Abscess. Beide Nierenbecken erweitert (Hydro. nephrose) mit verdunnter Rinde, in der zahlreiche narbige Einziehungen. Die Blasennarbe ist an den Bauchdecken adhärent, kegelförmig ausgezogen. Blasenhypertrophie. Bauchfell normal, keine Nebenverletzungen.

VII. W. G., 65 Jahre alt, Arbeiter aus Berlin. (1894. J.-Nr. 791.) 20. Juli bis 21. September 1894 .

Seit 8 Jahren Lungenkatarrh und Beginn der Blasenbeschwerden. Seit dem 19. Juli Harnverhaltung infolge starker Prostataschwellung. Herztöne sehr leise. Emphysem mit chronischem Bronchialkatarrl.

Urinirt seitdem nur durch den Dauerkatheter.

Am 31. Juli ist die Prostata halb so gross, allein es geht ein grangrtiner Fetzen nekrotisirter Schleimhant ab.

Am 10. September ist der Leib nirgends druckempfindlich, insbesondere kein Druckschmerz in der Nierengegend. Unter Fieber bildet sich eine Infiltration am Damm. Urin eiweissfrei, enthält Blasenepithelien, Eiter und Blutkörperchen, keine Bestandtheile eines Tumors.

Wegen Verdacht darauf wurde mit Verbrauch von 8,0 Chloroform und 30,0 Aether am 11. September die Cystostomie ohne Eröffnung des Bauchfelles gemacht, es findet sich jedoch ein Ventilverschluss durch Hypertrophie des prominenten mittleren Lappens der Prostata. Herausschälen von drei grösseren und einem kleineren Prostatamyom von der Blase aus, stumpf unter geringer Blutung. Einlage eines Nélatons in die Blase.

Erbrechen nach der Operation, jedoch kein Fieber mehr.

Nekrose der blossliegenden Fascia tranversa und des Peritoneums, während der Harn durch den Katheter abfliesst.

13. September. Leichte Blutung.

16. Sept. Abstossung der Nekrose, ohne jede Auftreibung des Bauches.

18. September. Bäder, Weissbier anf Wunsch, ohne dass Erbrechen wieder eintritt.

20. September. Blase zurückgesunken, nachdem die Nähte durcbgeschnitten haben.

21. September. Tod an zunehmender Erschöpfung.

Es fand sich Emphysem mit eitriger Bronchitis und bronchopneumonischen Herden im linken Unterlappen. Herz schlaff, braun, atrophisch. Klappen verdickt, Atherom der Aorta und besonders stark der Kranzarterien. Senile Nierenatropbie. Im Hirn Oedem und Tribnng der Pia, Atrophie der Gyri, geringe Erweiterung der Hirnventrikel. Atherom der linken Carotis interna. Die Bauchhöhle ist durch zwei an Stelle der Umschlagsialte adhärente Darmschlingen fest abgeschlossen, enthält blos normal glänzende, blasse Därme und keine Flüssigkeit. Die innere Wunde der Prostata sieht gut aus.

Die Castrationen wegen Prostatahypertrophie in Bethanien.

I. Wilhelm H. ist ohen mitgetheilt.

II. Fr. Sch., Hausdiener a us Berlin, $66 \mathrm{~J}$ abre alt. (1896. J.-Nr. 761.) 21. Juli bis 26. September 1896.

Tripper in der Jugend. Seit 14 Tagen Harnverhaltung, Epididymitis 
8 Tage lang, Cystitis, Urethritis ohne Gonokokken, durch seine grosse Unruhe beim Dauerkatheter.

Am 12. Angust wurde er auf seinen Wunsch, veranlasst durch $H$. (Nr. I), in meiner Vertretung von meinem Assistenten Herrn Dr. Sim on castrirt mit je einer Seidenligatur in toto und Verbrauch von 6,5 Chloroform. 20. September vernarbt.

26. September. Prostata nur noch zwei haselnussgrosse Knoten. Urinirt allein, im dinnen Strahl wegen einer noch nicht ganz behobenen Strictur.

III. R. H., Schneider, 66 Jahre alt, aus Berlin. (1896. J.-Nr. 677.) 21. Juni bis 3. November 1896.

Tripper in der Jugend.

Wimmernd vor Schmerz ist er wegen Blasentumors mit Hydrops des linken Beines ins Krankenhaus geschickt. Durch den Dauerkatheter wurde derselbe beseitigt. Obere Grenze der harten Prostata nicht abreichbar. Uriniren seitdem unmöglich.

24. Juli. Castration mit je einer Seidenligatur und 2 Drains durch Herrn Dr. Simon mit Verbrauch von 25,0 Chloroform.

Es erfolgt Phlebitis femoralis et poplitea sin. Ein Seidenfaden stösst sich links aus der Tiefe ab und verzögert die Heilung.

3. November. Prostata weich und kleiner, nur eine fingerbreite Schicht zwischen Katheter und Finger im Mastdarm. Urin und Uriniren normal.

IV. Fr. E., Postschaffuer, 78 Jahre alt, aus Berlin. (1896. J.-Nr. 993.) 1. October bis 3. November 1896.

Verheirathet. 1874 Tripper, 1886 Scrotalbruch links, 1894 auch rechts, 1850 das rechte Auge verloren durch einen Eisensplitter, Arteriosklerose der Arteria temporalis und radialis. Varicen am Unterschenkel.

Harnbeschwerden und Husten seit 3 Jahren, mit Harndrang in der letzten Zeit.

Prostata hart, obere Grenze nicht zu erreichen. Linker Lappen besonders gross, Harn trüb ohne Eiweiss.

20. October. Sectio aurea duplex mit $15 \mathrm{Grm}$. Chloroform und 57,5 Aether. Excision der Bruchsäcke. Naht der Bauchpforten, Fasciennaht und Hautnähte mit je 2 Drains. Die Gefässe der Stränge wurden einzeln mit Catgut unterbunden.

Puls darnach unregelmässig. Campher.

21. October. Drains fort.

27. October. Flockenlesen. Täglicher Verband wegen Urinausfluss auf den Verband trotz mehrmaligen Katheterns tăglich seit Entfernung des Dauerkatheters.

29. October. Entfernung aller Nähte. Blutausfluss aus der Drainstelle. Verwirrt, sehr unruhig. Senega.

Bei der Section fand sich in der linken Lungenspitze ein alter, ausgeheilter, tuberculöser Herd, Bronchitis und Hypostasen in den unteren I,appen. Arteriosklerose in der Aorta, leichte Verdickung an der Mitralis und an den Basillararterien. Linke Niere mit 2 Harnleitern, $65 \mathrm{Grm}$, $8 \mathrm{Cm}$. lang, $4 \mathrm{Cm}$. breit, $1 \frac{1 / 2}{2}-21 / 2 \mathrm{~cm}$ hoch, Rindensubstanz 2-3 Mm. Rechte Niere $175 \mathrm{Grm} ., 11 \times 6 \times 3 \frac{1}{1 / 2} \mathrm{Cm}$, Rinde $5-7 \frac{1}{1} 2 \mathrm{Mm}$. Faust- 
grosses Hämatom hinter dem rechten inneren Leistenring, Leber klein Pia trüb, Bauchfell normal.

V. August R., Pensionär, 71 Jahre alt, aus Berlin. (1897. J.-Nr. 175.) 9.-22. Januar 1897.

An einem doppelten Leistenbruch leidend, infolge der seit $1 \frac{1 / 2}{2}$ Jahren bestehenden Harnbeschwerden, hat er sich am 1. September 1896 eine ungeheilte intracapsulare Schenkelhalsfractur der rechten Seite zugezogen, welche ihm noch jetzt bei jeder leisen Bewegung sehr heftige Schmerzen machte und ihn seitdem zum Katheterleben verurtheilt hat. Ausserdem an chronischem Bronchialkatarrh leidend, war er infolge dieser vollständigen Harnverhaltung elend und benommen und doch dabei Nachts so unruhig, dass el oft vor Schmerz laut aufschrie.

Urin eitrig mit Eiweiss. Links wallnussgrose Hydrocele funiculi spermatici und Verdickung des linken Nebenhodens.

In der Hoffnung, seinem schweren Leiden vielleicht eine güostige Wendung dadurch geben zu können, machte ich ihm am 16. Januar mit $8 \mathrm{Grm}$. Chloroform und $15 \mathrm{Grm}$. Aether die Castration, wobei sich im linken Nebenhoden Eiter fand. Es trat zwar kein Erbrechen ein, allein bei seiner Unruhe trat am 18. Abends unter leichtem Fieber ein Hämatom im linken Scrotum durch Drainverstopfung ein.

Am Abend des 22. 39,4 und Tod unter zunehmender Benommenheit und beschleunigter Athmung.

Prostata in den 3 Lappen stark hypertrophisch, links ein kleinhaselnussgrosses Myom. Granularatrophie beider Nieren, Nierenarterienwände verdickt. In beiden unteren Lungenlappen Schluckpneumonie. Im Gehirn Pia trib, Atrophie der Gyri, hochgradige Arteriosklerose der Basilargefässe mit Kalkplatten. In der Blase zwischen den starken Trabekeln tiefe Eitertaschen.

VI. Paul St., 68 Jahre alt, Marmorschleifer aus Berlin. (1897. J.-Nr. 483.) 1. April bis 21. Mai 1897.

Er wurde Ende März mit rechtsseitiger Lungenentzlindung und vollständiger Harnverhaltung auf der inneren Station aufgenommen. Arteriosklerose. Rechts binten unten ging die Dämpfung bis zur Scapula, links hinten unten bestand Katarrh.

Die Harnbeschwerden haben sich seit 2 Jahren entwickelt, so dass er zuletzt am Tage alle $1 / 2$ Stunde, Nachts alle Stunden urinirte, oft unwillktrlich und fliessend. Prostata 3 Finger breit hoch und 4 breit. Urin ammoniakalisch.

Am 5. April hörte das Fieber auf. Nach Entfernung des Dauerkatheters wird kein Tropfen Urin, höchstens Stuhl herausgepresst, dann auch manchmal der Dauerkatheter.

Am 12. Mai rechts hinten unten vesiculäres Athmen, aber beiderseits noch hinten unten Rasseln. Sonst war er so gekräftigt, dass ich am 13. Mai ihm mit $10 \mathrm{Grm}$. Chloroform die Castration machte, mit 2 Catgutligaturen durch den Strang, und 3 Catgutnähten durch ihn und die Haut. Dabei wurde statt des Dauerkatheters regelmässiger Katheterismus angewendet. 
Am 17. urinirte er zum ersten Male wieder spontan, die Blase war aber nicht vollständig entleert.

Am 18. fand ich inn deshalb sehr froh, ohne Fieber und ohne Sticheiterung waren die Wunden verklebt und alle Fäden entfernt.

Leider ergab sich am 19. Abends $39,5^{\circ}$ und als Grund davon wieder eine rechtsseitige Lungenentzindung, die am 20. auch links hinten unten auftrat und am 21. zu vollständigem Kräfteverfall führte.

Bei der Section waren die Narben kaum noch zu erkennen. Der rechte Unterlappen und ein Theil des linken Unterlappens waren bepatisirt, oben Oedem. Cystitis mit vielen Eiterbuchten. Der Mittellappen ragt in die Blase. Kleine Fibromyome in der Prostata. Ureteren und Nierenbecken dilatirt, Ureteren voll Eiter. Nieren klein mit schmaler Rinde.

VII. Julius M., 59 Jahrealt, Eisenbahnwagenschmierer a us Berlin. (1897. J.-Nr.621.) 15. Mai 1897.

1870 Tripper und Pleuropneumonie. Vor $1 / 2$ Jahr Retention und seitdem kein Strahl. Wegen Harnverhaltung in der letzten Nacht vergeblicher Versuch des Katheterismus mit starker Blutung draussen. Bronchialkatarrh.

Bei der Aufnahme blutet er aus der Harnröhre und trotz der Narkose $(10,0 \mathrm{Grm}$. Chloroform) gelangt der Katheter stets in die falschen Wege. Obere Grenze der Prostata nicht abzureichen. Stand der Blase bis 3 Finger breit unter dem Nabel trotz eines warmen Bades.

15. Mai Nachts. Die Cystostomie (mit sofortiger Naht des oberhalb der tiefen Umschlagsfalte eröfneten Bauchfelles) ergiebt einen falschen Weg rechts wie links von der Urethra in der Prostata, welche, wieder in die klaffende innere Harnröhrenmündung von der Blase eingefuhrte Finger deutlich beim Metallkatheter fuhlen konnte, bis zur Blasenschleimbaut vordrangen. Naht der Bauchdecken bis zn den Nähten der Cystostomiewunde. Nach Entleerung der prallen Blase sofort Castration. Darnach hat sich die Harnröhrenmündung in der Blase vollständig zusammengezogen, so dass der retrograde Katheterismns sich nicht mit Leichtigkeit wollte ausführen lassen. Verbrauch von $15 \mathrm{Grm}$. Chloroform.

Am 18. Mai Drainage der Cystostomiewunde, so dass er von jetzt ab trocken liegt. Nach der Operation trat weder Erbrechen, noch Fieber ein.

Am 28. Mai wurde, nachdem beide Scrotalwunden prima intentione geheilt, ein Dauerkatheter eingefuhrt.

Am 3. Juni wurden statt des Katheters permanente Bäder angewendet, die zuletzt wieder mit dem Danerkatheter vertauscht wurden, weil sie den dicken Kranken angriffen.

Die Bauchwunde war Ende Juni geheilt. Allein im Juli entleerte sich noch ein Seidenfaden aus einem kleinen Abscess in ihr. Bei der Entlassung am 14. August war der Urin normal, die Bauchnarbe fest, und der dankbare Kranke ganz erholt. Der Katheter fühlte sich vom Mastdarm wie nackt an, so grundlich war die Prostata verschwunden.

Welch' Contrast zwischen diesen 3 Reihen von 7 Fällen. 